\title{
New approaches for the solution of space-time fractional Schrödinger equation
}

\author{
Ali Demir ${ }^{1 *}$, Mine Aylin Bayrak and Ebru Ozbilge ${ }^{2}$
}

"Correspondence:

ademir@kocaeli.edu.tr

'Department of Mathematics, Kocaeli University, Kocaeli, Turkey Full list of author information is available at the end of the article

\begin{abstract}
The aim of this study is to establish the solution of the time-space fractional Schrödinger equation subject to initial and boundary conditions which has many applications in science such as nonlinear optics, plasma physics, super conductivity, based on the residual power series method (RPSM). We first apply suitable transformations to make the order of one of the fractional derivatives integer to implement the RPSM easily to construct the fractional power series solution. The method proposed in this article gives highly encouraging results. Illustrative examples show that this method is compatible with solving such fractional differential equations.
\end{abstract}

Keywords: Fractional Schrödinger equation; Residual power series method; Space-time fractional differential equations

\section{Introduction}

Mathematical modeling is an undeniably powerful tool for systems since a mathematical description of processes allows us to figure out quantitative and qualitative behavior of it in analysis. Moreover, mathematical models involving a fractional order derivative lead to an excellent description for the properties of the behavior of nonlinear systems in various branches of Science and Engineering [1-7]. From this point of view, fractional order models provide better predictions than integer order models. Therefore, in recent decades, fractional order models have been used in a wide range of fields such as physics, chemistry, biology,engineering, optimal control theory, and finance [8-26]. Fractional order mathematical models have been recently employed for complex systems with memory and hereditary properties to provide deep understanding of the phenomena since fractional derivatives are non-local operators. It is worth while mentioning that selecting the type of fractional derivative is based on the experimental data to adjust the model to find the evolution of the phenomena with nonlinear behavior and memory. Since the mathematical models involving a Caputo fractional order derivative have classical initial conditions, the Caputo fractional derivative and its extensions are widely used to model systems in diverse areas of sciences. Moreover, the Caputo fractional derivative of a constant is zero unlike the other fractional derivatives. As a result, fractional order models in the Caputo sense are developed to be able to study the complex behavior of real evolution processes

(c) The Author(s) 2020. This article is licensed under a Creative Commons Attribution 4.0 International License, which permits use sharing, adaptation, distribution and reproduction in any medium or format, as long as you give appropriate credit to the original author(s) and the source, provide a link to the Creative Commons licence, and indicate if changes were made. The images or other third party material in this article are included in the article's Creative Commons licence, unless indicated otherwise in a credit line to the material. If material is not included in the article's Creative Commons licence and your intended use is not permitted by statutory regulation or exceeds the permitted use, you will need to obtain permission directly from the copyright holder. To view a copy of this licence, visit http://creativecommons.org/licenses/by/4.0/. 
with memory and hereditary properties much better. Besides modeling, developing reliable analytical methods to solve fractional differential equations is an emerging area since finding exact solutions of many fractional differential equations is hard. Hence, considerable attention has been given to utilizing new powerful and efficient methods and software programs to obtain analytical and accurate numerical solutions.

The fractional Schrödinger equation (SE) plays an important role in fractional quantum mechanics [27-30]. Since the beginning the solution of a space-time SE is a significant topic of physical, mathematical and engineering research, in this article the solution of it is constructed based on the method so-called RPSM which is a well-known analytic technique, with a new transformation [31]. Since the behavior of real world systems is affected by their historical states, modeling them via fractional PDEs is recommended to understand and analyze real world systems. Therefore fractional PDEs have drawn the attention of many scientists in various research areas [32-35]. In many cases obtaining the solution of a space-time SE analytically is not always possible, and that is why solving them by numerical methods is very common [35-37].

The main purpose of the present study is to establish the space-time SE by using RPSM and some new transformations. The main advantage of these new transformations is in reducing the space-time SE to either time SE or space SE for which applying RPSM is easier.

In the present article, a new transformation is constructed to implement RPSM to obtain approximately the solution for the following space-time fractional SE general dimensionless form:

$$
\begin{aligned}
& i D_{t}^{\alpha} u+\delta D_{x}^{\beta+1} u+\gamma|u(x, t)|^{2} u(x, t)+\phi(x) u(x, t)=0, \\
& u\left(x, t_{0}\right)=\varphi(x), \\
& u\left(x_{0}, t\right)=\mu_{1}(t), \\
& u_{x}\left(x_{0}, t\right)=\mu_{2}(t),
\end{aligned}
$$

where $x, \delta, \gamma \in R, t \geq t_{0}, 0<\alpha, \beta \leq 1, i^{2}=-1$, and $|\cdot|$ is the modulus. Here, $u(x, t), \phi(x)$ and $\varphi(x)$ represent the macroscopic wave function, the external trapping potential analytic function and an analytic function, respectively. This mathematical and physical model has various applications in science such as nonlinear optics, plasma physics, superconductivity, and quantum mechanics [27-30, 38-43].

\section{Preliminaries}

In this section properties of fractional calculus theory which allow us to construct the solution of space-time fractional SE are presented [8]. We first give the main definitions and various features of the fractional calculus theory in this section. The Riemann-Liouville fractional integral operator of order $\alpha(\alpha \geq 0)$ is defined as

$$
\begin{aligned}
& J^{\alpha} f(x)=\frac{1}{\Gamma(\alpha)} \int_{0}^{x}(x-t)^{\alpha-1} f(t) d t, \quad \alpha>0, x>0, \\
& J^{0} f(x)=f(x) .
\end{aligned}
$$


The Caputo fractional derivative of order $\alpha$ is defined as

$$
\begin{aligned}
& D^{\alpha} f(x)=J^{m-\alpha} D^{m} f(x)=\frac{1}{\Gamma(m-\alpha)} \int_{0}^{x}(x-t)^{m-\alpha-1} f^{(m)}(t) d t, \\
& m-1<\alpha \leq m, \quad x>0,
\end{aligned}
$$

where $D^{m}$ is the classical differential operator of order $m$.

Let $n$ be the smallest integer greater than $\alpha$, the time fractional derivative operator of order $\alpha$ of $u(x, t)$ is defined as [8]

$$
D_{t}^{\alpha} u(x, t)=\frac{\partial^{\alpha} u(x, t)}{\partial t^{\alpha}}= \begin{cases}\frac{1}{\Gamma(n-\alpha)} \int_{0}^{t}(t-\tau)^{n-\alpha-1} \frac{\partial^{n} u(\tau, t)}{\partial t^{n}} d \tau, & n-1<\alpha \leq n, \\ \frac{\partial^{n} u(x, t)}{\partial t^{n}}, & \alpha=n \in N .\end{cases}
$$

If $n-1<\alpha \leq n, u(x, t) \in C_{\mu}^{n}, n \in N$ and $\mu \geq-1$ then $D_{t}^{\alpha} J_{t}^{\alpha} u(x, t)=u(x, t)$ and $J_{t}^{\alpha} D_{t}^{\alpha} u(x, t)=$ $u(x, t)-\sum_{j=0}^{n-1} \frac{\partial^{j} u\left(x, s^{+}\right)}{\partial t^{j}} \frac{(t-s)^{j}}{j !}$, where $t>s \geq 0$. The power series expansions about $t=t_{0}$ and $x=x_{0}$,

$$
\sum_{k=0}^{\infty} \sum_{l=0}^{n-1} f_{k l}(x)\left(t-t_{0}\right)^{k \alpha+l}, \quad 0 \leq n-1<\alpha \leq n, t_{0} \leq t<t_{0}+R
$$

and

$$
\sum_{k=0}^{\infty} \sum_{l=0}^{m-1} g_{k l}(t)\left(x-x_{0}\right)^{k \beta+l}, \quad 0 \leq m-1<\beta \leq m, x_{0} \leq x<x_{0}+R,
$$

are called multiple fractional power series, where $f_{k l}(x)$ and $g_{k l}(t)$ are called the coefficients of the series.

\section{Solution for space-time fractional SE based on RPSM}

In order to solve space-time fractional SE (1)-(4) by RPSM, the problem is first reduced to either a space fractional SE or time fractional SE, which leads to the following cases.

Case 1: Simplification of the space-time fractional SE via the transformation $u=I_{x}^{\beta-1} v$

To get rid of the space fractional derivative in Eq. (1) the transformation $u=I_{x}^{\beta-1} v$ is taken into account. As a result the following problem is obtained:

$$
\begin{aligned}
& i D_{t}^{\alpha}\left(I_{x}^{\beta-1} v\right)+\delta v_{x x}+\gamma\left|I_{x}^{\beta-1} v\right|^{2} I_{x}^{\beta-1} v+\phi(x) I_{x}^{\beta-1} v=0, \\
& v\left(x, t_{0}\right)=I_{x}^{1-\beta} \varphi(x) \\
& v\left(x_{0}, t\right)=0 \\
& v_{x}\left(x_{0}, t\right)=0 .
\end{aligned}
$$

Now, the RPSM is implemented to construct multiple fractional power series solution subject to initial condition. To establish the approximate solution the real and imaginary parts of the function $v(x, t)$ and the initial condition $I_{x}^{1-\beta} \varphi(x)$ can be rewritten as follows:

$$
v(x, t)=w(x, t)+i z(x, t), v\left(x, t_{0}\right)=I_{x}^{1-\beta} w_{0}(x, t)+i I_{x}^{1-\beta} z_{0}(x, t),
$$




$$
\begin{aligned}
& i\left[D_{t}^{\alpha}\left(I_{x}^{\beta-1} w\right)+\delta z_{x x}+\gamma\left(\left(I_{x}^{\beta-1} w\right)^{2}+\left(I_{x}^{\beta-1} z\right)^{2}\right) I_{x}^{\beta-1} z+\phi(x) I_{x}^{\beta-1} z\right] \\
& \quad-\left[D_{t}^{\alpha}\left(I_{x}^{\beta-1} z\right)-\delta w_{x x}-\gamma\left(\left(I_{x}^{\beta-1} w\right)^{2}+\left(I_{x}^{\beta-1} z\right)^{2}\right) I_{x}^{\beta-1} w-\phi(x) I_{x}^{\beta-1} w\right]=0,
\end{aligned}
$$

while the initial condition of Eq. (12) can be separated in the following form:

$$
v\left(x, t_{0}\right)=I_{x}^{1-\beta} w_{0}(x, t)+i I_{x}^{1-\beta} z_{0}(x, t)=f_{0}(x)+i g_{0}(x) .
$$

According to the results of Eqs. (15), (16) and (17) the space-time fractional SE can be converted into an equivalent system of PDEs as follows:

$$
\begin{aligned}
& D_{t}^{\alpha}\left(I_{x}^{\beta-1} w\right)+\delta z_{x x}+\gamma\left(\left(I_{x}^{\beta-1} w\right)^{2}+\left(I_{x}^{\beta-1} z\right)^{2}\right) I_{x}^{\beta-1} z+\phi(x) I_{x}^{\beta-1} z=0, \\
& D_{t}^{\alpha}\left(I_{x}^{\beta-1} z\right)-\delta w_{x x}-\gamma\left(\left(I_{x}^{\beta-1} w\right)^{2}+\left(I_{x}^{\beta-1} z\right)^{2}\right) I_{x}^{\beta-1} w-\phi(x) I_{x}^{\beta-1} w=0,
\end{aligned}
$$

based on the initial conditions

$$
\begin{aligned}
& w\left(x, t_{0}\right)=f_{0}(x), \\
& z\left(x, t_{0}\right)=g_{0}(x) .
\end{aligned}
$$

Let $w_{k}(x, t)$ and $z_{k}(x, t)$ be defined as follows:

$$
\begin{aligned}
& w_{k}(x, t)=\sum_{j=0}^{k} f_{j}(x) \frac{\left(t-t_{0}\right)^{j \alpha}}{\Gamma(1+j \alpha)}, \\
& z_{k}(x, t)=\sum_{j=0}^{k} g_{j}(x) \frac{\left(t-t_{0}\right)^{j \alpha}}{\Gamma(1+j \alpha)},
\end{aligned}
$$

which are called the $k$ th truncated series of $w(x, t)$ and $z(x, t)$. It is clear that the conditions $w\left(x, t_{0}\right)=f_{0}(x)$ and $z\left(x, t_{0}\right)=g_{0}(x)$ hold. To determine the coefficients $f_{j}(x)$ and $g_{j}(x), j=$ $1,2,3, \ldots, k$, in Eqs. (20), the residual functions are defined as follows:

$$
\begin{aligned}
& \operatorname{Res}^{1}(x, t)=D_{t}^{\alpha}\left(I_{x}^{\beta-1} w\right)+\delta z_{x x}+\gamma\left(\left(I_{x}^{\beta-1} w\right)^{2}+\left(I_{x}^{\beta-1} z\right)^{2}\right) I_{x}^{\beta-1} z+\phi(x) I_{x}^{\beta-1} z, \\
& \operatorname{Res}^{2}(x, t)=D_{t}^{\alpha}\left(I_{x}^{\beta-1} z\right)-\delta w_{x x}-\gamma\left(\left(I_{x}^{\beta-1} w\right)^{2}+\left(I_{x}^{\beta-1} z\right)^{2}\right) I_{x}^{\beta-1} w-\phi(x) I_{x}^{\beta-1} w .
\end{aligned}
$$

Hence the $k$ th truncated residual functions are obtained in the following form:

$$
\begin{aligned}
& \operatorname{Res}_{k}^{1}(x, t)=D_{t}^{\alpha}\left(I_{x}^{\beta-1} w_{k}\right)+\delta\left(z_{k}\right)_{x x}+\gamma\left(\left(I_{x}^{\beta-1} w_{k}\right)^{2}+\left(I_{x}^{\beta-1} z_{k}\right)^{2}\right) I_{x}^{\beta-1} z_{k}+\phi(x) I_{x}^{\beta-1} z_{k}, \\
& \operatorname{Res}_{k}^{2}(x, t)=D_{t}^{\alpha}\left(I_{x}^{\beta-1} z_{k}\right)-\delta\left(w_{k}\right)_{x x}-\gamma\left(\left(I_{x}^{\beta-1} w_{k}\right)^{2}+\left(I_{x}^{\beta-1} z_{k}\right)^{2}\right) I_{x}^{\beta-1} w_{k}-\phi(x) I_{x}^{\beta-1} w_{k} .
\end{aligned}
$$

Equating the equation including $D_{t}^{(n-1) \alpha}$ of $\operatorname{Res}_{j}^{1}(x, t)$ and $\operatorname{Res}_{j}^{2}(x, t), j=1,2,3, \ldots, k$, in Eqs. (22) to zero the following algebraic system is obtained:

$$
\begin{aligned}
& D_{t}^{(j-1) \alpha} \operatorname{Res}_{j}^{1}\left(x, t_{0}\right)=0, \quad j=1,2,3, \ldots, k, \\
& D_{t}^{(j-1) \alpha} \operatorname{Res}_{j}^{1}\left(x, t_{0}\right)=0, \quad j=1,2,3, \ldots, k .
\end{aligned}
$$


In order to determine the coefficients of $f_{1}(x)$ and $g_{1}(x)$ in Eq. (20), the truncated series $w_{1}(x, t)$ and $z_{1}(x, t)$ are plugged into the first truncated residual functions to obtain

$$
\begin{aligned}
& \operatorname{Res}_{1}^{1}(x, t)=D_{t}^{\alpha}\left(I_{x}^{\beta-1} w_{1}\right)+\delta\left(z_{1}\right)_{x x}+\gamma\left(\left(I_{x}^{\beta-1} w_{1}\right)^{2}+\left(I_{x}^{\beta-1} z_{1}\right)^{2}\right) I_{x}^{\beta-1} z_{1}+\phi(x) I_{x}^{\beta-1} z_{1}, \\
& \operatorname{Res}_{1}^{2}(x, t)=D_{t}^{\alpha}\left(I_{x}^{\beta-1} z_{1}\right)-\delta\left(w_{1}\right)_{x x}-\gamma\left(\left(I_{x}^{\beta-1} w_{1}\right)^{2}+\left(I_{x}^{\beta-1} z_{1}\right)^{2}\right) I_{x}^{\beta-1} w_{1}-\phi(x) I_{x}^{\beta-1} w_{1},
\end{aligned}
$$

But since $w_{1}(x, t)=f_{0}(x)+f_{1}(x) \frac{\left(t-t_{0}\right)^{\alpha}}{\Gamma(1+\alpha)}$ and $z_{1}(x, t)=g_{0}(x)+g_{1}(x) \frac{\left(t-t_{0}\right)^{\alpha}}{\Gamma(1+\alpha)}$, Eq. (24) leads to the following results:

$$
\begin{aligned}
\operatorname{Res}_{1}^{1}(x, t)= & I_{x}^{\beta-1} f_{1}(x)+\delta\left(g_{0}^{\prime \prime}(x)+g_{1}^{\prime \prime}(x) \frac{\left(t-t_{0}\right)^{\alpha}}{\Gamma(1+\alpha)}\right) \\
& +\gamma\left(\left(I_{x}^{\beta-1}\left(f_{0}(x)+f_{1}(x) \frac{\left(t-t_{0}\right)^{\alpha}}{\Gamma(1+\alpha)}\right)\right)^{2}\right. \\
& \left.+\left(I_{x}^{\beta-1}\left(g_{0}(x)+g_{1}(x) \frac{\left(t-t_{0}\right)^{\alpha}}{\Gamma(1+\alpha)}\right)\right)^{2}\right) \\
& \times I_{x}^{\beta-1}\left(g_{0}(x)+g_{1}(x) \frac{\left(t-t_{0}\right)^{\alpha}}{\Gamma(1+\alpha)}\right) \\
& +\phi(x) I_{x}^{\beta-1}\left(g_{0}(x)+g_{1}(x) \frac{\left(t-t_{0}\right)^{\alpha}}{\Gamma(1+\alpha)}\right), \\
\operatorname{Res}_{2}^{1}(x, t)= & I_{x}^{\beta-1} g_{1}(x)-\delta\left(f_{0}^{\prime \prime}(x)+f_{1}^{\prime \prime}(x) \frac{\left(t-t_{0}\right)^{\alpha}}{\Gamma(1+\alpha)}\right) \\
& -\gamma\left(\left(I_{x}^{\beta-1}\left(g_{0}(x)+g_{1}(x) \frac{\left(t-t_{0}\right)^{\alpha}}{\Gamma(1+\alpha)}\right)\right)^{2}\right. \\
& \left.+\left(I_{x}^{\beta-1}\left(f_{0}(x)+f_{1}(x) \frac{\left(t-t_{0}\right)^{\alpha}}{\Gamma(1+\alpha)}\right)\right)^{2}\right) \\
& \times I_{x}^{\beta-1}\left(f_{0}(x)+f_{1}(x) \frac{\left(t-t_{0}\right)^{\alpha}}{\Gamma(1+\alpha)}\right) \\
& -\phi(x) I_{x}^{\beta-1}\left(f_{0}(x)+f_{1}(x) \frac{\left(t-t_{0}\right)^{\alpha}}{\Gamma(1+\alpha)}\right),
\end{aligned}
$$

Substituting of $t=t_{0}$ in Eq. (25) leads to the following:

$$
\begin{aligned}
& I_{x}^{\beta-1} f_{1}(x)=-\delta g_{0^{\prime \prime}}-\gamma\left(\left(I_{x}^{\beta-1} f_{0}\right)^{2}+\left(I_{x}^{\beta-1} g_{0}\right)^{2}\right) I_{x}^{\beta-1} g_{0}-\phi(x) I_{x}^{\beta-1} g_{0}, \\
& I_{x}^{\beta-1} g_{1}(x)=\delta f_{0^{\prime \prime}}+\gamma\left(\left(I_{x}^{\beta-1} f_{0}\right)^{2}+\left(I_{x}^{\beta-1} g_{0}\right)^{2}\right) I_{x}^{\beta-1} f_{0}+\phi(x) I_{x}^{\beta-1} f_{0} .
\end{aligned}
$$

In a similar way, the unknown coefficients $f_{2}(x)$ and $g_{2}(x)$ are computed by substituting $w_{2}(x, t)=f_{0}(x)+f_{1}(x) \frac{\left(t-t_{0}\right)^{\alpha}}{\Gamma(1+\alpha)}+f_{2}(x) \frac{\left(t-t_{0}\right)^{2 \alpha}}{\Gamma(1+2 \alpha)}$ and $z_{2}(x, t)=g_{0}(x)+g_{1}(x) \frac{\left(t-t_{0}\right)^{\alpha}}{\Gamma(1+\alpha)}+g_{2}(x) \frac{\left(t-t_{0}\right)^{2 \alpha}}{\Gamma(1+2 \alpha)}$ into the second truncated residual functions $\operatorname{Res}_{2}^{1}(x, t)$ and $\operatorname{Res}_{2}^{2}(x, t)$ of Eq. (22) so we have

$$
\begin{aligned}
\operatorname{Res}_{1}^{2}(x, t)= & I_{x}^{\beta-1}\left(f_{1}(x)+f_{2}(x) \frac{\left(t-t_{0}\right)^{\alpha}}{\Gamma(1+\alpha)}\right) \\
& +\delta\left(g_{0}^{\prime \prime}(x)+g_{1}^{\prime \prime}(x) \frac{\left(t-t_{0}\right)^{\alpha}}{\Gamma(1+\alpha)}+g_{2}^{\prime \prime}(x) \frac{\left(t-t_{0}\right)^{2 \alpha}}{\Gamma(1+2 \alpha)}\right) \\
& +\gamma\left(\left(I_{x}^{\beta-1}\left(f_{0}(x)+f_{1}(x) \frac{\left(t-t_{0}\right)^{\alpha}}{\Gamma(1+\alpha)}+f_{2}(x) \frac{\left(t-t_{0}\right)^{2 \alpha}}{\Gamma(1+2 \alpha)}\right)\right)^{2}\right.
\end{aligned}
$$




$$
\begin{aligned}
& \left.+\left(I_{x}^{\beta-1}\left(g_{0}(x)+g_{1}(x) \frac{\left(t-t_{0}\right)^{\alpha}}{\Gamma(1+\alpha)}+g_{2}(x) \frac{\left(t-t_{0}\right)^{2 \alpha}}{\Gamma(1+2 \alpha)}\right)\right)^{2}\right) \\
& \times I_{x}^{\beta-1}\left(g_{0}(x)+g_{1}(x) \frac{\left(t-t_{0}\right)^{\alpha}}{\Gamma(1+\alpha)}+g_{2}(x) \frac{\left(t-t_{0}\right)^{2 \alpha}}{\Gamma(1+2 \alpha)}\right) \\
& +\phi(x) I_{x}^{\beta-1}\left(g_{0}(x)+g_{1}(x) \frac{\left(t-t_{0}\right)^{\alpha}}{\Gamma(1+\alpha)}+g_{2}(x) \frac{\left(t-t_{0}\right)^{2 \alpha}}{\Gamma(1+2 \alpha)}\right) \\
\operatorname{Res}_{2}^{2}(x, t)= & I_{x}^{\beta-1}\left(g_{1}(x)+g_{2}(x) \frac{\left(t-t_{0}\right)^{\alpha}}{\Gamma(1+\alpha)}\right) \\
& -\delta\left(f_{0}^{\prime \prime}(x)+f_{1}^{\prime \prime}(x) \frac{\left(t-t_{0}\right)^{\alpha}}{\Gamma(1+\alpha)}+f_{2}^{\prime \prime}(x) \frac{\left(t-t_{0}\right)^{2 \alpha}}{\Gamma(1+2 \alpha)}\right) \\
& -\gamma\left(\left(I_{x}^{\beta-1}\left(g_{0}(x)+g_{1}(x) \frac{\left(t-t_{0}\right)^{\alpha}}{\Gamma(1+\alpha)}+g_{2}(x) \frac{\left(t-t_{0}\right)^{2 \alpha}}{\Gamma(1+2 \alpha)}\right)\right)^{2}\right. \\
& \left.+\left(I_{x}^{\beta-1}\left(f_{0}(x)+f_{1}(x) \frac{\left(t-t_{0}\right)^{\alpha}}{\Gamma(1+\alpha)}+f_{2}(x) \frac{\left(t-t_{0}\right)^{2 \alpha}}{\Gamma(1+2 \alpha)}\right)\right)^{2}\right) \\
& \times I_{x}^{\beta-1}\left(f_{0}(x)+f_{1}(x) \frac{\left(t-t_{0}\right)^{\alpha}}{\Gamma(1+\alpha)}+f_{2}(x) \frac{\left(t-t_{0}\right)^{2 \alpha}}{\Gamma(1+2 \alpha)}\right) \\
& \phi(x) I_{x}^{\beta-1}\left(f_{0}(x)+f_{1}(x) \frac{\left(t-t_{0}\right)^{\alpha}}{\Gamma(1+\alpha)}+f_{2}(x) \frac{\left(t-t_{0}\right)^{2 \alpha}}{\Gamma(1+2 \alpha)}\right) \\
& \\
& \\
\Gamma(1+\alpha) & \\
\Gamma(x) &
\end{aligned}
$$

Now, applying the operator $D_{t}^{\alpha}$ and substituting of $t=t_{0}$, solving the resultant system for $f_{2}(x)$ and $g_{2}(x)$ one gets

$$
\begin{aligned}
I_{x}^{\beta-1} f_{2}(x)= & -\delta g_{1}^{\prime \prime}-2 \gamma I_{x}^{\beta-1} f_{0} I_{x}^{\beta-1} g_{0} I_{x}^{\beta-1} f_{1}-\gamma\left(\left(I_{x}^{\beta-1} f_{0}\right)^{2}\right) I_{x}^{\beta-1} g_{1} \\
& -3 \gamma\left(\left(I_{x}^{\beta-1} g_{0}\right)^{2}\right) I_{x}^{\beta-1} g_{1}-\phi(x) I_{x}^{\beta-1} g_{1}, \\
I_{x}^{\beta-1} g_{2}(x)= & \delta f_{1}^{\prime \prime}+2 \gamma I_{x}^{\beta-1} g_{0} I_{x}^{\beta-1} f_{0} I_{x}^{\beta-1} g_{1}+\gamma\left(\left(I_{x}^{\beta-1} g_{0}\right)^{2}\right) I_{x}^{\beta-1} f_{1} \\
& +3 \gamma\left(\left(I_{x}^{\beta-1} f_{0}\right)^{2}\right) I_{x}^{\beta-1} f_{1}+\phi(x) I_{x}^{\beta-1} f_{1} .
\end{aligned}
$$

As before, the same procedure for $j=3$ is applied to construct the following $I_{x}^{\beta-1} f_{3}, I_{x}^{\beta-1} g_{3}$ :

$$
\begin{aligned}
I_{x}^{\beta-1} f_{3}(x)= & -\delta g_{2}^{\prime \prime}-\phi(x) I_{x}^{\beta-1} g_{2} \\
& -\gamma\left[2 I_{x}^{\beta-1} f_{0} I_{x}^{\beta-1} g_{0} I_{x}^{\beta-1} f_{2}+\gamma\left(\left(I_{x}^{\beta-1} f_{0}\right)^{2}\right) I_{x}^{\beta-1} g_{2}+3 \gamma\left(\left(I_{x}^{\beta-1} g_{0}\right)^{2}\right) I_{x}^{\beta-1} g_{2}\right] \\
& -\gamma\left[2 I_{x}^{\beta-1} f_{0} I_{x}^{\beta-1} g_{1} I_{x}^{\beta-1} f_{1}+\left(\left(I_{x}^{\beta-1} f_{1}\right)^{2}\right) I_{x}^{\beta-1} g_{0}+3\left(\left(I_{x}^{\beta-1} g_{1}\right)^{2}\right) I_{x}^{\beta-1} g_{0}\right] \\
& \times \frac{\Gamma(1+2 \alpha)}{\Gamma(1+\alpha)^{2}}, \\
I_{x}^{\beta-1} g_{3}(x)= & f_{2}^{\prime \prime}+\phi(x) I_{x}^{\beta-1} f_{2} \\
& +\gamma\left[2 I_{x}^{\beta-1} g_{0} I_{x}^{\beta-1} f_{0} I_{x}^{\beta-1} g_{2}+\gamma\left(\left(I_{x}^{\beta-1} g_{0}\right)^{2}\right) I_{x}^{\beta-1} f_{2}+3 \gamma\left(\left(I_{x}^{\beta-1} f_{0}\right)^{2}\right) I_{x}^{\beta-1} f_{2}\right] \\
& +\gamma\left[2 I_{x}^{\beta-1} g_{0} I_{x}^{\beta-1} g_{1} I_{x}^{\beta-1} f_{1}+\left(\left(I_{x}^{\beta-1} g_{1}\right)^{2}\right) I_{x}^{\beta-1} f_{0}+3\left(\left(I_{x}^{\beta-1} g_{1}\right)^{2}\right) I_{x}^{\beta-1} g_{0}\right] \\
& \times \frac{\Gamma(1+2 \alpha)}{\Gamma(1+\alpha)^{2}} .
\end{aligned}
$$


The recurrence relation among the coefficients of the multiple fractional power series solution for space-time fractional SE is constructed by repeating this procedure:

$$
\begin{aligned}
u(x, t)= & \varphi(x)+\left(I_{x}^{\beta-1} f_{1} \frac{\left(t-t_{0}\right)^{\alpha}}{\Gamma(1+\alpha)}+I_{x}^{\beta-1} f_{2} \frac{\left(t-t_{0}\right)^{2 \alpha}}{\Gamma(1+2 \alpha)}\right. \\
& \left.+I_{x}^{\beta-1} f_{3} \frac{\left(t-t_{0}\right)^{3 \alpha}}{\Gamma(1+3 \alpha)}+\cdots\right)+i\left(I_{x}^{\beta-1} g_{1} \frac{\left(t-t_{0}\right)^{\alpha}}{\Gamma(1+\alpha)}\right. \\
& \left.+I_{x}^{\beta-1} g_{2} \frac{\left(t-t_{0}\right)^{2 \alpha}}{\Gamma(1+2 \alpha)}+I_{x}^{\beta-1} g_{3} \frac{\left(t-t_{0}\right)^{3 \alpha}}{\Gamma(1+3 \alpha)}+\cdots\right),
\end{aligned}
$$

which is equivalent to the $j$ th truncated series of $u(x, t)$; that is,

$$
u_{k}(x, t)=\sum_{j=0}^{k} \varphi_{j}(x) \frac{\left(t-t_{0}\right)^{j \alpha}}{\Gamma(1+j \alpha)}
$$

Case 2: Simplification of the space-time fractional SE via the transformation $u=I_{t}^{\alpha-1} v$

To get rid of the time fractional derivative in Eq. (1) the transformation $u=I_{t}^{\alpha-1} v$ is taken into account. As a result the following problem is obtained:

$$
\begin{aligned}
& i v_{t}+\delta D_{x}^{\beta+1}\left(I_{t}^{\alpha-1} v\right)+\gamma\left|I_{t}^{\alpha-1} v\right|^{2} I_{t}^{\alpha-1} v+\phi(x) I_{t}^{\alpha-1} v=0, \\
& v\left(x, t_{0}\right)=0, \\
& v\left(x_{0}, t\right)=I_{t}^{1-\alpha} \mu_{1}(t), \\
& v_{x}\left(x_{0}, t\right)=I_{t}^{1-\alpha} \mu_{2}(t) .
\end{aligned}
$$

Now, the RPSM is implemented to construct a multiple fractional power series solution subject to boundary conditions. To establish the approximate solution the real and imaginary parts of the function $v(x, t)$ and the initial condition $I_{t}^{1-\alpha} \varphi(x)$ can be rewritten as follows:

$$
\begin{aligned}
& v(x, t)=w(x, t)+i z(x, t), v_{0}(x, t)=v\left(x_{0}, t\right)+\left(x-x_{0}\right) v_{x}\left(x_{0}, t\right)=w_{0}(x, t)+i z_{0}(x, t), \\
& i\left[w_{t}+\delta D_{x}^{\beta+1}\left(I_{t}^{\alpha-1} z\right)+\gamma\left(\left(I_{t}^{\alpha-1} w\right)^{2}+\left(I_{t}^{\alpha-1} z\right)^{2}\right) I_{t}^{\alpha-1} z+\phi(x) I_{t}^{\alpha-1} z\right] \\
& \quad-\left[z_{t}-\delta D_{x}^{\beta+1}\left(I_{t}^{\alpha-1} w\right)-\gamma\left(\left(I_{t}^{\alpha-1} w\right)^{2}+\left(I_{t}^{\alpha-1} z\right)^{2}\right) I_{t}^{\alpha-1} w-\phi(x) I_{t}^{\alpha-1} w\right]=0,
\end{aligned}
$$

while the initial condition of Eq. (36) can be separated in the following form:

$$
v_{0}(x, t)=I_{x}^{1-\beta} w_{0}(x, t)+i I_{x}^{1-\beta} z_{0}(x, t)=f_{0}(t)+\left(x-x_{0}\right) f_{1}(t)+i\left(g_{0}(t)+\left(x-x_{0}\right) g_{1}(t)\right) .
$$

According to the results of Eqs. (36), (37) and (38) the space-time fractional SE can be converted into an equivalent system of PDEs as follows:

$$
\begin{aligned}
& w_{t}+\delta D_{x}^{\beta+1}\left(I_{t}^{\alpha-1} z\right)+\gamma\left(\left(I_{t}^{\alpha-1} w\right)^{2}+\left(I_{t}^{\alpha-1} z\right)^{2}\right) I_{t}^{\alpha-1} z+\phi(x) I_{t}^{\alpha-1} z=0, \\
& z_{t}-\delta D_{x}^{\beta+1}\left(I_{t}^{\alpha-1} w\right)-\gamma\left(\left(I_{t}^{\alpha-1} w\right)^{2}+\left(I_{t}^{\alpha-1} z\right)^{2}\right) I_{t}^{\alpha-1} w-\phi(x) I_{t}^{\alpha-1} w=0
\end{aligned}
$$


based on boundary conditions:

$$
\begin{aligned}
& w_{0}(x, t)=f_{0}(t)+\left(x-x_{0}\right) f_{1}(t), \\
& z_{0}(x, t)=g_{0}(t)+\left(x-x_{0}\right) g_{1}(t) .
\end{aligned}
$$

To determine the coefficients $f_{j}(t)$ and $g_{j}(t), j=1,2,3, \ldots, k$, in Eqs. (39), the residual functions are defined as follows:

$$
\begin{aligned}
& \operatorname{Res}^{1}(x, t)=w_{t}+\delta D_{x}^{\beta+1}\left(I_{t}^{\alpha-1}\right) z+\gamma\left(\left(I_{t}^{\alpha-1} w\right)^{2}+\left(I_{t}^{\alpha-1} z\right)^{2}\right) I_{t}^{\alpha-1} z+\phi(x) I_{t}^{\alpha-1} z, \\
& \operatorname{Res}^{2}(x, t)=z_{t}-\delta D_{x}^{\beta+1}\left(I_{t}^{\alpha-1}\right) w-\gamma\left(\left(I_{t}^{\alpha-1} w\right)^{2}+\left(I_{t}^{\alpha-1} z\right)^{2}\right) I_{t}^{\alpha-1} w-\phi(x) I_{t}^{\alpha-1} w,
\end{aligned}
$$

and the $k$ th truncated residual functions are

$$
\begin{aligned}
& \operatorname{Res}_{k}^{1}=\left(w_{k}\right)_{t}+\delta D_{x}^{\beta+1}\left(I_{t}^{\alpha-1}\right) z_{k}+\gamma\left(\left(I_{t}^{\alpha-1} w_{k}\right)^{2}+\left(I_{t}^{\alpha-1} z_{k}\right)^{2}\right) I_{t}^{\alpha-1} z_{k}+\phi(x) I_{t}^{\alpha-1} z_{k} \\
& \operatorname{Res}_{k}^{2}=\left(z_{k}\right)_{t}-\delta D_{x}^{\beta+1}\left(I_{t}^{\alpha-1}\right) w_{k}-\gamma\left(\left(I_{t}^{\alpha-1} w_{k}\right)^{2}+\left(I_{t}^{\alpha-1} z_{k}\right)^{2}\right) I_{t}^{\alpha-1} w_{k}-\phi(x) I_{t}^{\alpha-1} w_{k} .
\end{aligned}
$$

In order to determine the coefficients of $f_{1}(x)$ and $g_{1}(x)$ in Eq. (20), the truncated series $w_{1}(x, t)$ and $z_{1}(x, t)$ are plugged into the first truncated residual functions to obtain

$$
\begin{aligned}
& \operatorname{Res}_{1}^{1}=\left(w_{1}\right)_{t}+\delta D_{x}^{\beta+1}\left(I_{t}^{\alpha-1}\right) z_{1}+\gamma\left(\left(I_{t}^{\alpha-1} w_{1}\right)^{2}+\left(I_{t}^{\alpha-1} z_{1}\right)^{2}\right) I_{t}^{\alpha-1} z_{1}+\phi(x) I_{t}^{\alpha-1} z_{1} \\
& \operatorname{Res}_{1}^{2}=\left(z_{1}\right)_{t}-\delta D_{x}^{\beta+1}\left(I_{t}^{\alpha-1}\right) w_{1}-\gamma\left(\left(I_{t}^{\alpha-1} w_{1}\right)^{2}+\left(I_{t}^{\alpha-1} z_{1}\right)^{2}\right) I_{t}^{\alpha-1} w_{1}-\phi(x) I_{t}^{\alpha-1} w_{1} .
\end{aligned}
$$

But since $w_{1}(x, t)=f_{0}(t)+f_{1}(t)\left(x-x_{0}\right)+f_{2}(t) \frac{\left(x-x_{0}\right)^{\beta+1}}{\Gamma(2+\beta)}$ and $z_{1}(x, t)=g_{0}(t)+g_{1}(t)\left(x-x_{0}\right)+$ $g_{2}(t) \frac{\left(x-x_{0}\right)^{\beta+1}}{\Gamma(2+\beta)}$, Eq. (43) leads to the following results:

$$
\begin{aligned}
\operatorname{Res}_{1}^{1}(x, t)= & f_{0}^{\prime}(t)+f_{1}^{\prime}(t)\left(x-x_{0}\right)+f_{2}^{\prime}(t) \frac{\left(x-x_{0}\right)^{\beta+1}}{\Gamma(2+\beta)}+\delta I_{t}^{\alpha-1} g_{2}(t) \\
& +\gamma\left(\left(I_{t}^{\alpha-1}\left(f_{0}(t)+f_{1}(t)\left(x-x_{0}\right)+f_{2}(t) \frac{\left(x-x_{0}\right)^{\beta+1}}{\Gamma(2+\beta)}\right)\right)^{2}\right. \\
& \left.+\left(I_{t}^{\alpha-1}\left(g_{0}(t)+g_{1}(t)\left(x-x_{0}\right)+g_{2}(t) \frac{\left(x-x_{0}\right)^{\beta+1}}{\Gamma(2+\beta)}\right)\right)^{2}\right) \\
& \times I_{t}^{\alpha-1}\left(g_{0}(t)+g_{1}(t)\left(x-x_{0}\right)+g_{2}(t) \frac{\left(x-x_{0}\right)^{\beta+1}}{\Gamma(2+\beta)}\right) \\
& +\phi(x) I_{t}^{\alpha-1}\left(g_{0}(t)+g_{1}(t)\left(x-x_{0}\right)+g_{2}(t) \frac{\left(x-x_{0}\right)^{\beta+1}}{\Gamma(2+\beta)}\right) \\
\operatorname{Res}_{1}^{2}(x, t)= & g_{0}^{\prime}(t)+g_{1}^{\prime}(t)\left(x-x_{0}\right)+g_{2}^{\prime}(t) \frac{\left(x-x_{0}\right)^{\beta+1}}{\Gamma(2+\beta)}-\delta I_{t}^{\alpha-1} f_{2}(t) \\
& -\gamma\left(\left(I_{t}^{\alpha-1}\left(f_{0}(t)+f_{1}(t)\left(x-x_{0}\right)+f_{2}(t) \frac{\left(x-x_{0}\right)^{\beta+1}}{\Gamma(2+\beta)}\right)\right)^{2}\right. \\
& \left.+\left(I_{t}^{\alpha-1}\left(g_{0}(t)+g_{1}(t)\left(x-x_{0}\right)+g_{2}(t) \frac{\left(x-x_{0}\right)^{\beta+1}}{\Gamma(2+\beta)}\right)\right)^{2}\right) \\
& \times I_{t}^{\alpha-1}\left(f_{0}(t)+f_{1}(t)\left(x-x_{0}\right)+f_{2}(t) \frac{\left(x-x_{0}\right)^{\beta+1}}{\Gamma(2+\beta)}\right)
\end{aligned}
$$




$$
-\phi(x) I_{t}^{\alpha-1}\left(f_{0}(t)+f_{1}(t)\left(x-x_{0}\right)+f_{2}(t) \frac{\left(x-x_{0}\right)^{\beta+1}}{\Gamma(2+\beta)}\right) .
$$

Substituting of $x=x_{0}$ in Eq. (44) leads to the following:

$$
\begin{aligned}
& I_{t}^{\alpha-1} g_{2}(t)=\left[-f_{0^{\prime}}-\gamma\left(\left(I_{t}^{\alpha-1} f_{0}\right)^{2}+\left(I_{t}^{\alpha-1} g_{0}\right)^{2}\right) I_{t}^{\alpha-1} g_{0}-\phi(x) I_{t}^{\alpha-1} g_{0}\right] / \delta \\
& I_{t}^{\alpha-1} f_{2}(t)=\left[g_{0^{\prime \prime}}-\gamma\left(\left(I_{t}^{\alpha-1} f_{0}\right)^{2}+\left(I_{t}^{\alpha-1} g_{0}\right)^{2}\right) I_{t}^{\alpha-1} f_{0}-\phi(x) I_{t}^{\alpha-1} f_{0}\right] / \delta
\end{aligned}
$$

In a similar way, the unknown coefficients $f_{3}(t)$ and $g_{3}(t)$ are computed by substituting $w_{3}(x, t)=f_{0}(t)+f_{1}(t)\left(x-x_{0}\right)+f_{2}(t) \frac{\left(x-x_{0}\right)^{\beta+1}}{\Gamma(2+\beta)}+f_{3}(t) \frac{\left(x-x_{0}\right)^{\beta+2}}{\Gamma(3+\beta)}$ and $z_{3}(x, t)=g_{0}(t)+g_{1}(t)\left(x-x_{0}\right)+$ $g_{2}(t) \frac{\left(x-x_{0}\right)^{\beta+1}}{\Gamma(2+\beta)}+g_{3}(t) \frac{\left(x-x_{0}\right)^{\beta+2}}{\Gamma(3+\beta)}$ into the second truncated residual functions, $\operatorname{Res}_{2}^{1}(x, t)$ and $\operatorname{Res}_{2}^{2}(x, t)$, of Eq. (45) to have

$$
\begin{aligned}
& \operatorname{Res}_{1}^{2}(x, t)=f_{0}^{\prime}(t)+f_{1}^{\prime}(t)\left(x-x_{0}\right)+f_{2}^{\prime}(t) \frac{\left(x-x_{0}\right)^{\beta+1}}{\Gamma(2+\beta)}+f_{3}^{\prime}(t) \frac{\left(x-x_{0}\right)^{\beta+2}}{\Gamma(3+\beta)} \\
& +\delta I_{t}^{\alpha-1}\left(g_{2}(t)+g_{3}(t)\left(x-x_{0}\right)\right) \\
& +\gamma\left(\left(I_{t}^{\alpha-1}\left(f_{0}(t)+f_{1}(t)\left(x-x_{0}\right)+f_{2}(t) \frac{\left(x-x_{0}\right)^{\beta+1}}{\Gamma(2+\beta)}+f_{3}(t) \frac{\left(x-x_{0}\right)^{\beta+2}}{\Gamma(3+\beta)}\right)\right)^{2}\right. \\
& \left.+\left(I_{t}^{\alpha-1}\left(g_{0}(t)+g_{1}(t)\left(x-x_{0}\right)+g_{2}(t) \frac{\left(x-x_{0}\right)^{\beta+1}}{\Gamma(2+\beta)}+g_{3}(t) \frac{\left(x-x_{0}\right)^{\beta+2}}{\Gamma(3+\beta)}\right)\right)^{2}\right) \\
& \times I_{t}^{\alpha-1}\left(g_{0}(t)+g_{1}(t) x+g_{2}(t) \frac{x^{\beta+1}}{\Gamma(2+\beta)}+g_{3}(t) \frac{x^{\beta+2}}{\Gamma(3+\beta)}\right) \\
& +\phi(x) I_{t}^{\alpha-1}\left(g_{0}(t)+g_{1}(t)\left(x-x_{0}\right)+g_{2}(t) \frac{\left(x-x_{0}\right)^{\beta+1}}{\Gamma(2+\beta)}+g_{3}(t) \frac{\left(x-x_{0}\right)^{\beta+2}}{\Gamma(3+\beta)}\right), \\
& \operatorname{Res}_{2}^{2}(x, t)=g_{0}^{\prime}(t)+g_{1}^{\prime}(t)\left(x-x_{0}\right)+g_{2}^{\prime}(t) \frac{\left(x-x_{0}\right)^{\beta+1}}{\Gamma(2+\beta)}+g_{3}^{\prime}(t) \frac{\left(x-x_{0}\right)^{\beta+2}}{\Gamma(3+\beta)} \\
& -\delta I_{t}^{\alpha-1}\left(f_{2}(t)+f_{3}(t)\left(x-x_{0}\right)\right) \\
& -\gamma\left(\left(I_{t}^{\alpha-1}\left(f_{0}(t)+f_{1}(t)\left(x-x_{0}\right)+f_{2}(t) \frac{\left(x-x_{0}\right)^{\beta+1}}{\Gamma(2+\beta)}+f_{3}(t) \frac{\left(x-x_{0}\right)^{\beta+2}}{\Gamma(3+\beta)}\right)\right)^{2}\right. \\
& \left.+\left(I_{t}^{\alpha-1}\left(g_{0}(t)+g_{1}(t)\left(x-x_{0}\right)+g_{2}(t) \frac{\left(x-x_{0}\right)^{\beta+1}}{\Gamma(2+\beta)}+g_{3}(t) \frac{\left(x-x_{0}\right)^{\beta+2}}{\Gamma(3+\beta)}\right)\right)^{2}\right) \\
& \times I_{t}^{\alpha-1}\left(f_{0}(t)+f_{1}(t)\left(x-x_{0}\right)+f_{2}(t) \frac{\left(x-x_{0}\right)^{\beta+1}}{\Gamma(2+\beta)}+f_{3}(t) \frac{\left(x-x_{0}\right)^{\beta+2}}{\Gamma(3+\beta)}\right) \\
& -\phi(x) I_{t}^{\alpha-1}\left(f_{0}(t)+f_{1}(t)\left(x-x_{0}\right)+f_{2}(t) \frac{\left(x-x_{0}\right)^{\beta+1}}{\Gamma(2+\beta)}+f_{3}(t) \frac{\left(x-x_{0}\right)^{\beta+2}}{\Gamma(3+\beta)}\right) \text {. }
\end{aligned}
$$

Now, applying the operator $D_{x}$ and substituting of $x=x_{0}$ as follows:

$$
\begin{aligned}
I_{t}^{\alpha-1} g_{3}(t)= & {\left[-f_{1}^{\prime}-\gamma\left[3\left(I_{t}^{\alpha-1} g_{0}\right)^{2} I_{t}^{\alpha-1} g_{1}+2\left(I_{t}^{\alpha-1} f_{0} I_{t}^{\alpha-1} f_{1} I_{t}^{\alpha-1} g_{0}+\left(I_{t}^{\alpha-1} f_{0}\right)^{2} I_{t}^{\alpha-1} g_{1}\right]\right.\right.} \\
& \left.-\phi(x) I_{t}^{\alpha-1} g_{1}\right] / \delta \\
I_{t}^{\alpha-1} f_{3}(t)= & -g_{1}^{\prime}-\gamma\left[3\left(I_{t}^{\alpha-1} f_{0}\right)^{2} I_{t}^{\alpha-1} f_{1}+2\left(I_{t}^{\alpha-1} f_{0} I_{t}^{\alpha-1} g_{1} I_{t}^{\alpha-1} g_{0}+\left(I_{t}^{\alpha-1} g_{0}\right)^{2} I_{t}^{\alpha-1} f_{1}\right]\right. \\
& \left.-\phi(x) I_{t}^{\alpha-1} g_{1}\right] / \delta .
\end{aligned}
$$


The recurrence relation among the coefficients of the multiple fractional power series solution for space-time fractional SE is constructed by repeating this procedure,

$$
\begin{aligned}
u(x, t)= & \mu_{1}(t)+\mu_{2}(t)\left(x-x_{0}\right)+\left(I_{t}^{\alpha-1} f_{2} \frac{\left(x-x_{0}\right)^{\beta+1}}{\Gamma(2+\beta)}+I_{t}^{\alpha-1} f_{3} \frac{\left(x-x_{0}\right)^{\beta+2}}{\Gamma(3+\beta)}+\cdots\right) \\
& +i\left(I_{t}^{\alpha-1} g_{2} \frac{\left(x-x_{0}\right)^{\beta+1}}{\Gamma(2+\beta)}+I_{t}^{\alpha-1} g_{3} \frac{\left(x-x_{0}\right)^{\beta+2}}{\Gamma(3+\beta)}+\cdots\right)
\end{aligned}
$$

which is equivalent to the $j$ th truncated series of $u(x, t)$; that is,

$$
u_{k}(x, t)=\mu_{1}(t)+\mu_{2}(t)\left(x-x_{0}\right)+\sum_{j=3}^{k} \mu_{j}(t) \frac{\left(x-x_{0}\right)^{\beta+j-2}}{\Gamma(j-1+\beta)} .
$$

\section{Numerical examples}

This section is devoted to the following illustrative examples.

Example 1 Let us consider the problem including a space-time fractional SE

$$
\begin{aligned}
& i D_{t}^{\alpha} u-D_{x}^{\beta+1} u=0, \\
& u(x, 0)=e^{3 i x}, \\
& u(0, t)=e^{9 i t}, \\
& u_{x}(0, t)=3 i e^{9 i t},
\end{aligned}
$$

Case 1: Eqs. (50)-(53) transform as follows:

$$
\begin{aligned}
& i D_{t}^{\alpha}\left(I_{x}^{\beta-1} v\right)-v_{x x}=0, \\
& v(x, 0)=I_{x}^{1-\beta} e^{3 i x}, \\
& v(0, t)=0, \\
& v_{x}(0, t)=0 .
\end{aligned}
$$

To establish the approximate solution, Eqs. (54)-(55) are converted into an equivalent system of the space-time fractional SE via $u(x, t)=w(x, t)+i z(x, t)$ as follows:

$$
\begin{aligned}
& D_{t}^{\alpha}\left(I_{x}^{\beta-1} w\right)-z_{x x}=0, \\
& D_{t}^{\alpha}\left(I_{x}^{\beta-1} z\right)-w_{x x}=0,
\end{aligned}
$$

with the initial conditions

$$
\begin{aligned}
& w(x, 0)=I_{x}^{1-\beta} \cos (3 x)=3^{\beta-1} \cos \left(3 x+\frac{\pi}{2}(\beta-1)\right), \\
& z(x, 0)=I_{x}^{1-\beta} \sin (3 x)=3^{\beta-1} \sin \left(3 x+\frac{\pi}{2}(\beta-1)\right) .
\end{aligned}
$$

Here, $\delta=1, \gamma=1, \phi(x)=0, f_{0}(x)=I_{x}^{1-\beta} \cos (3 x), g_{0}(x)=I_{x}^{1-\beta} \sin (3 x)$. The unknown coefficients $I_{x}^{\beta-1} f_{j}, I_{x}^{\beta-1} g_{j}, j=0,1,2,3$, are computed via the initial approximations $w_{0}(x, t)=$ 
$I_{x}^{1-\beta} \cos (3 x), z_{0}(x, t)=I_{x}^{1-\beta} \sin (3 x)$ and RPSM. We have

$$
\begin{aligned}
& I_{x}^{\beta-1} f_{0}(x)=\cos (3 x), \\
& I_{x}^{\beta-1} f_{1}(x)=D_{x}^{\beta+1} \sin (3 x)=\frac{9 i}{2} x^{1-\beta}\left[E_{1,2-\beta}(3 i x)-E_{1,2-\beta}(-3 i x)\right], \\
& I_{x}^{\beta-1} f_{2}(x)=-D_{x}^{2 \beta+2} \cos (3 x)=\frac{-81}{2} x^{2-2 \beta}\left[E_{1,3-2 \beta}(3 i x)+E_{1,3-2 \beta}(-3 i x)\right], \\
& I_{x}^{\beta-1} f_{3}(x)=-D_{x}^{3 \beta+3} \sin (3 x)=\frac{-729 i}{2} x^{3-3 \beta}\left[E_{1,4-3 \beta}(3 i x)-E_{1,4-3 \beta}(-3 i x)\right], \\
& I_{x}^{\beta-1} g_{0}(x)=\sin (3 x), \\
& I_{x}^{\beta-1} g_{1}(x)=-D_{x}^{\beta+1} \cos (3 x)=\frac{9}{2} x^{1-\beta}\left[E_{1,2-\beta}(3 i x)+E_{1,2-\beta}(-3 i x)\right], \\
& I_{x}^{\beta-1} g_{2}(x)=-D_{x}^{2 \beta+2} \sin (3 x)=\frac{81 i}{2} x^{2-2 \beta}\left[E_{1,3-2 \beta}(3 i x)-E_{1,3-2 \beta}(-3 i x)\right], \\
& I_{x}^{\beta-1} g_{3}(x)=D_{x}^{3 \beta+3} \cos (3 x)=\frac{-729}{2} x^{3-3 \beta}\left[E_{1,4-3 \beta}(3 i x)+E_{1,4-3 \beta}(-3 i x)\right] .
\end{aligned}
$$

The third order RPS solutions can be constructed as follows:

$$
\begin{aligned}
w_{3}(x, t)= & \cos (3 x)+D_{x}^{\beta+1} \sin (3 x) \frac{t^{\alpha}}{\Gamma(1+\alpha)}-D_{x}^{2 \beta+2} \cos (3 x) \frac{t^{2 \alpha}}{\Gamma(1+2 \alpha)} \\
& -D_{x}^{3 \beta+3} \sin (3 x) \frac{t^{3 \alpha}}{\Gamma(1+3 \alpha)}, \\
z_{3}(x, t)= & \sin (3 x)-D_{x}^{\beta+1} \cos (3 x) \frac{t^{\alpha}}{\Gamma(1+\alpha)}-D_{x}^{2 \beta+2} \sin (3 x) \frac{t^{2 \alpha}}{\Gamma(1+2 \alpha)} \\
& +D_{x}^{3 \beta+3} \cos (3 x) \frac{t^{3 \alpha}}{\Gamma(1+3 \alpha)} .
\end{aligned}
$$

By making some algebraic properties of complex numbers, the general pattern coinciding with the exact solution can be established as follows:

$$
\begin{aligned}
u(x, t)= & e^{3 i x}+i D_{x}^{\beta+1} e^{3 i x} \frac{t^{\alpha}}{\Gamma(1+\alpha)}+i^{2} D_{x}^{2 \beta+2} e^{3 i x} \frac{t^{2 \alpha}}{\Gamma(1+2 \alpha)} \\
& +i^{3} D_{x}^{3 \beta+3} e^{3 i x} \frac{t^{3 \alpha}}{\Gamma(1+3 \alpha)}+\cdots
\end{aligned}
$$

Case 2: Eqs. (50)-(53) transform as follows:

$$
\begin{aligned}
& i v_{t}-D_{x}^{\beta+1} I_{t}^{\alpha-1} v=0, \\
& v(x, 0)=0, \\
& v(0, t)=I_{t}^{1-\alpha} e^{9 i t}, \\
& v_{x}(0, t)=3 i I_{t}^{1-\alpha} e^{9 i t} .
\end{aligned}
$$


To construct the approximate solution, from Eq. (36), Eqs. (64)-(67) can be converted into an equivalent system of PDEs as follows:

$$
\begin{aligned}
& w_{t}-D_{x}^{\beta+1} I_{t}^{\alpha-1} z=0, \\
& z_{t}-D_{x}^{\beta+1} I_{t}^{\alpha-1} w=0,
\end{aligned}
$$

subject to the boundary conditions

$$
\begin{aligned}
& w_{0}(x, t)=I_{t}^{1-\alpha} \cos (9 t)-3 x I_{t}^{1-\alpha} \sin (9 t), \\
& z_{0}(x, t)=I_{t}^{1-\alpha} \sin (9 t)+3 x I_{t}^{1-\alpha} \cos (9 t) .
\end{aligned}
$$

Here, $\delta=1, \gamma=1, \phi(x)=0, f_{0}(x)=I_{t}^{1-\alpha} \cos (9 t), f_{1}(x)=-3 I_{t}^{1-\alpha} \sin (9 t)$ and $g_{0}(x)=I_{t}^{1-\alpha} \sin (9 t)$, $g_{1}(x)=3 I_{t}^{1-\alpha} \cos (9 t)$. Anyhow, using RPS method, starting with the initial guesses approximations $w_{0}(x, t)=I_{t}^{1-\alpha} \cos (9 t)-3 x I_{t}^{1-\alpha} \sin (9 t)$ and $z_{0}(x, t)=I_{t}^{1-\alpha} \sin (9 t)+3 x I_{t}^{1-\alpha} \cos (9 t)$ with the $k$ th truncated residual functions of Eq. (58) is used when $j=3$ throughout the computations; the following forms for the unknown coefficients $I_{t}^{\alpha-1} f_{j}, I_{t}^{\alpha-1} g_{j}, j=0,1,2,3$, are obtained:

$$
\begin{aligned}
& I_{t}^{\alpha-1} f_{0}(t)=\cos (9 t), \\
& I^{\alpha-1} f_{1}(t)=-3 \sin (9 t), \\
& I_{t}^{\alpha-1} f_{2}(t)=-D_{t}^{\alpha} \sin (9 t)=\frac{-9}{2} t^{1-\alpha}\left[E_{1,2-\alpha}(9 i t)+E_{1,2-\alpha}(-9 i t)\right], \\
& I_{t}^{\alpha-1} f_{3}(t)=-3 D_{t}^{\alpha} \cos (9 t)=\frac{-27 i}{2} t^{1-\alpha}\left[E_{1,2-\alpha}(9 i t)-E_{1,2-\alpha}(-9 i t)\right], \\
& I_{t}^{\alpha-1} g_{0}(t)=\sin (9 t), \\
& I_{t}^{\alpha-1} g_{1}(t)=3 \cos (9 t), \\
& I_{t}^{\alpha-1} g_{2}(t)=D_{t}^{\alpha} \cos (9 t)=\frac{9 i}{2} t^{1-\alpha}\left[E_{1,2-\alpha}(9 i t)-E_{1,2-\alpha}(-9 i t)\right], \\
& I_{t}^{\alpha-1} g_{3}(t)=-3 D_{t}^{\alpha} \sin (9 t)=\frac{-27}{2} t^{1-\alpha}\left[E_{1,2-\alpha}(9 i t)+E_{1,2-\alpha}(-9 i t)\right] .
\end{aligned}
$$

The third order RPS solutions can be constructed as follows:

$$
\begin{aligned}
w_{3}(x, t)= & \cos (9 t)-3 x \sin (9 t)-D_{t}^{\alpha} \sin (9 t) \frac{x^{\beta+1}}{\Gamma(2+\beta)} \\
& -3 D_{t}^{\alpha} \cos (9 t) \frac{x^{\beta+2}}{\Gamma(3+\beta)}, \\
z_{3}(x, t)= & \sin (9 t)+3 x \cos (9 t)+D_{t}^{\alpha} \cos (9 t) \frac{x^{\beta+1}}{\Gamma(2+\beta)} \\
& -3 D_{t}^{\alpha} \sin (9 t) \frac{x^{\beta+2}}{\Gamma(3+\beta)}
\end{aligned}
$$




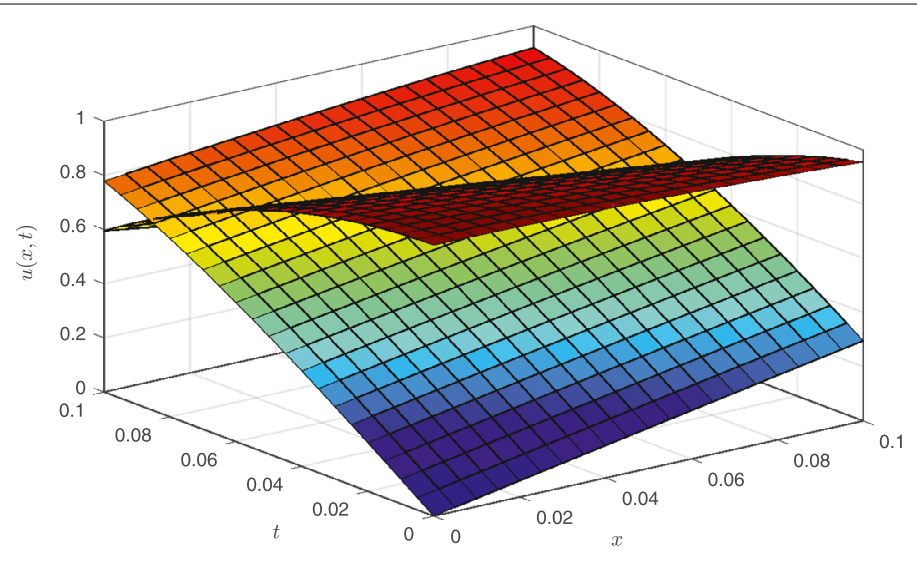

Figure 1 The approximate solution of Example 1 for Case 1 and $\alpha=\beta=1$

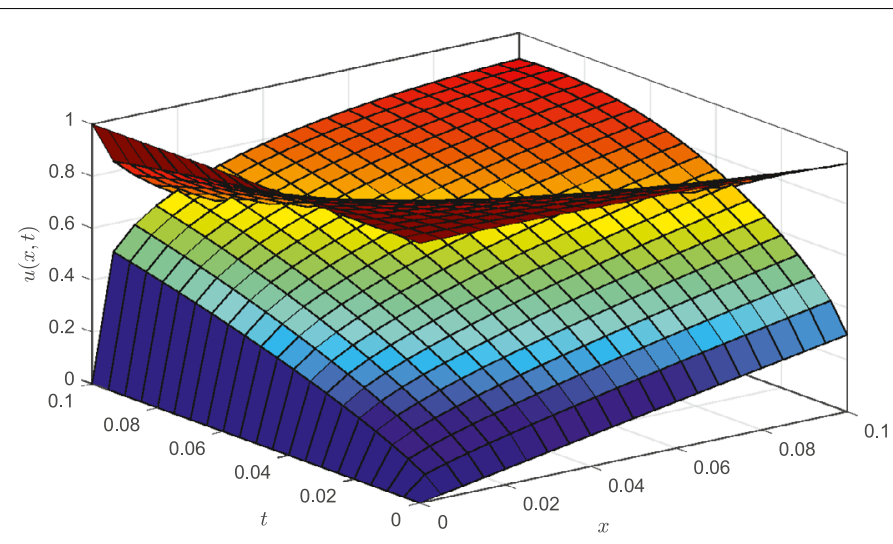

Figure 2 The approximate solution of Example 1 for Case 1 and $\alpha=\beta=0.75$

By making some algebraic properties of complex numbers, the general pattern form coinciding with the exact solution can be established as follows:

$$
u(x, t)=e^{9 i t}+3 x e^{9 i t}+i D_{t}^{\alpha} e^{9 i t} \frac{x^{\beta+1}}{\Gamma(2+\beta)}+3 i^{2} D_{t}^{\alpha} e^{9 i t} \frac{x^{\beta+2}}{\Gamma(3+\beta)}+\cdots
$$

It is clear from Figs. 1-6, the approximate solutions of Example 1 for case 1 and case 2 for different orders of fractional derivatives give better results for small values $x$ and $t$.

Example 2 Let us consider the problem including space-time fractional SE

$$
\begin{aligned}
& i D_{t}^{\alpha} u+D_{x}^{\beta+1} u-2|u|^{2} u=0, \\
& u(x, 0)=e^{i x} \\
& u(0, t)=e^{-3 i t} \\
& u_{x}(0, t)=i e^{-3 i t}
\end{aligned}
$$




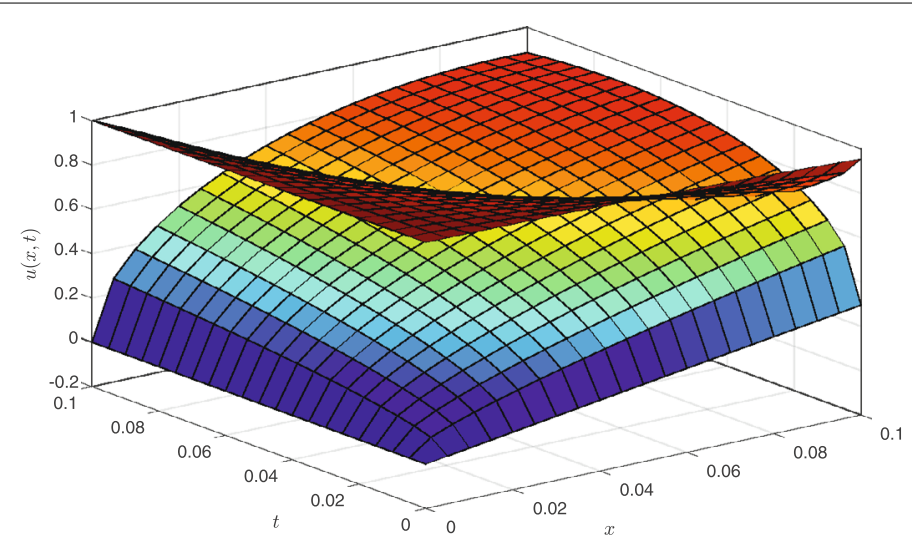

Figure 3 The approximate solution of Example 1 for Case 1 and $\alpha=\beta=0.5$

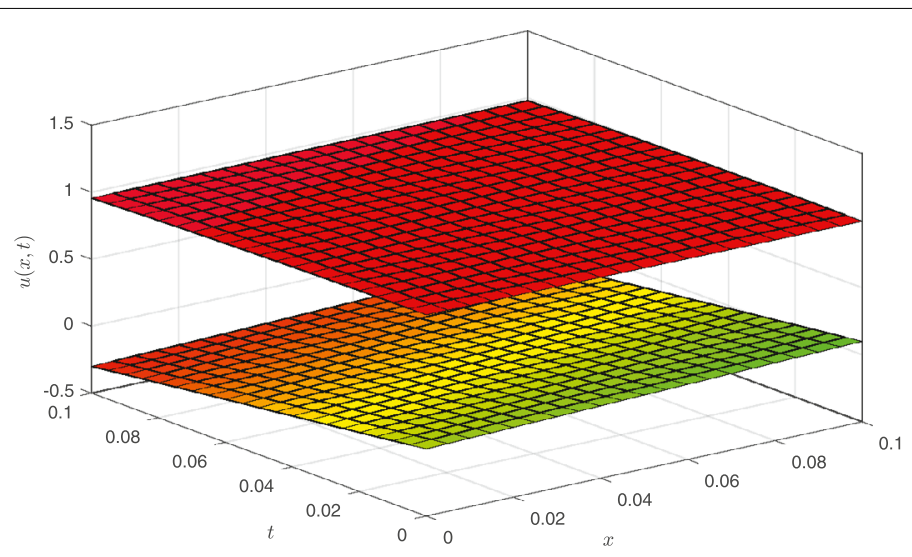

Figure 4 The approximate solution of Example 1 for Case 2 and $\alpha=\beta=1$

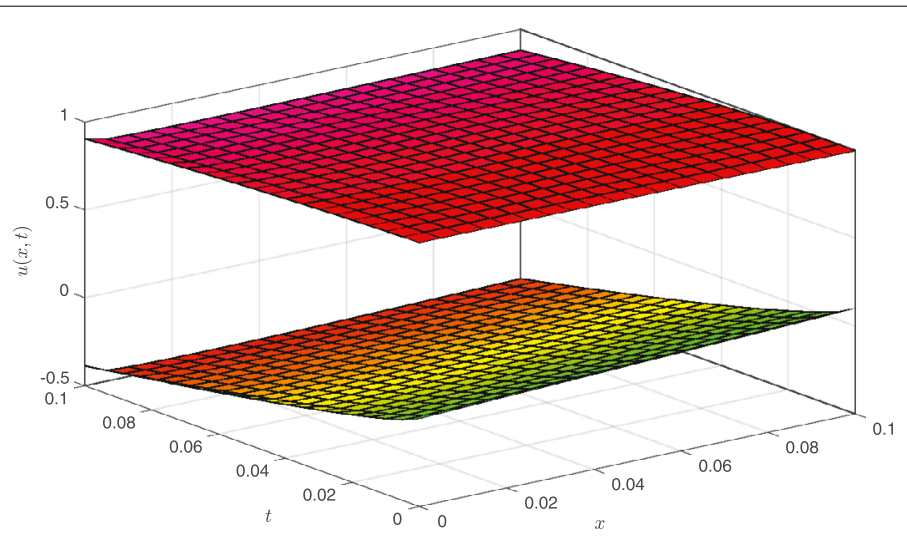

Figure 5 The approximate solution of Example 1 for Case 2 and $\alpha=\beta=0.75$

Case 1: Eqs. (74)-(77) transform as follows:

$$
i D_{t}^{\alpha}\left(I_{x}^{\beta-1} v\right)+v_{x x}-2\left|I_{x}^{\beta-1} v\right|^{2} I_{x}^{\beta-1} v=0
$$




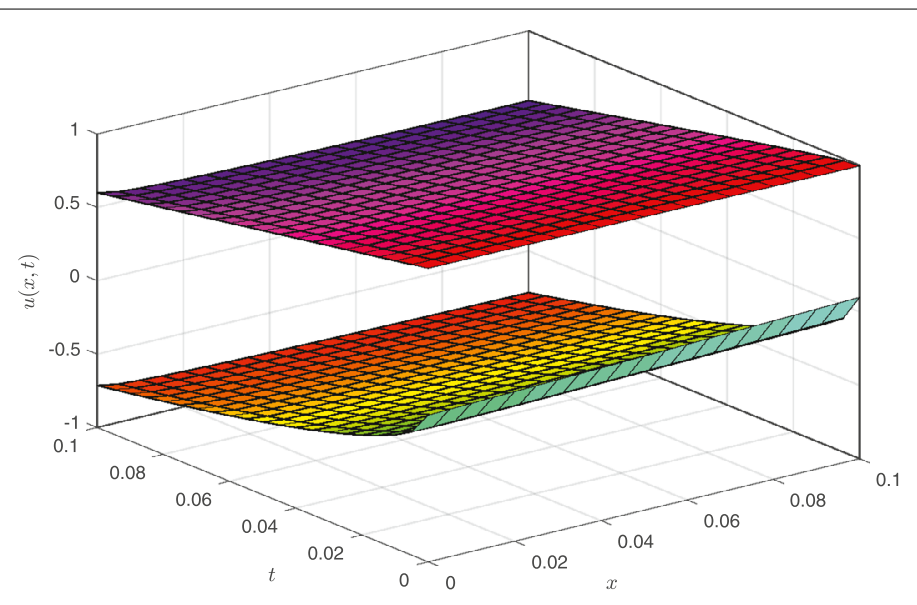

Figure 6 The approximate solution of Example 1 for Case 2 and $\alpha=\beta=0.5$

$$
\begin{aligned}
& v(x, 0)=I_{x}^{1-\beta} e^{i x}, \\
& v(0, t)=0, \\
& v_{x}(0, t)=0 .
\end{aligned}
$$

To establish the approximate solution, Eqs. (78)-(79) are converted into an equivalent system of space-time fractional SE via $u(x, t)=w(x, t)+i z(x, t)$ as follows:

$$
\begin{aligned}
& D_{t}^{\alpha}\left(I_{x}^{\beta-1} w\right)+z_{x x}-2\left[\left(I_{x}^{\beta-1} w\right)^{2}+\left(I_{x}^{\beta-1} z\right)^{2}\right] I_{x}^{\beta-1} z=0, \\
& D_{t}^{\alpha}\left(I_{x}^{\beta-1} z\right)-w_{x x}+2\left[\left(I_{x}^{\beta-1} w\right)^{2}+\left(I_{x}^{\beta-1} z\right)^{2}\right] I_{x}^{\beta-1} w=0,
\end{aligned}
$$

with the initial conditions

$$
\begin{aligned}
& w(x, 0)=I_{x}^{1-\beta} \cos (x)=\cos \left(x+\frac{\pi}{2}(\beta-1)\right), \\
& z(x, 0)=I_{x}^{1-\beta} \sin (x)=\sin \left(x+\frac{\pi}{2}(\beta-1)\right) .
\end{aligned}
$$

Here $\delta=1, \gamma=2, \phi(x)=0, f_{0}(x)=I_{x}^{1-\beta} \cos (x), g_{0}(x)=I_{x}^{1-\beta} \sin (x)$. The unknown coefficients $I_{x}^{\beta-1} f_{j}, I_{x}^{\beta-1} g_{j}, j=0,1,2$, are computed via the initial approximations $w_{0}(x, t)=I_{x}^{1-\beta} \cos (x)$ and $z_{0}(x, t)=I_{x}^{1-\beta} \sin (x)$ and RPSM. We have

$$
\begin{aligned}
I_{x}^{\beta-1} f_{0}(x)= & \cos (x), \\
I_{x}^{\beta-1} f_{1}(x)= & -D_{x}^{\beta+1} \sin (x)+2 \sin (x), \\
I_{x}^{\beta-1} f_{2}(x)= & -\left(D_{x}^{2 \beta+2} \cos (x)-2 D_{x}^{\beta+1} \cos (x)\right) \\
& +4 \sin (x) \cos (x)\left(-D_{x}^{\beta+1} \sin (x)+2 \sin (x)\right) \\
& +\left(2 \cos ^{2}(x)+6 \sin ^{2}(x)\right)\left(D_{x}^{\beta+1} \cos (x)-2 \cos (x)\right),
\end{aligned}
$$




$$
\begin{aligned}
I_{x}^{\beta-1} f_{0}(x)= & \sin (x), \\
I_{x}^{\beta-1} f_{1}(x)= & D_{x}^{\beta+1} \cos (x)-2 \cos (x), \\
I_{x}^{\beta-1} f_{2}(x)= & -D_{x}^{2 \beta+2} \sin (x)+2 D_{x}^{\beta+1} \sin (x)-4 \sin (x) \cos (x)\left(D_{x}^{\beta+1} \cos (x)-2 \cos (x)\right) \\
& -\left(6 \cos ^{2}(x)+2 \sin ^{2}(x)\right)\left(-D_{x}^{\beta+1} \sin (x)+2 \sin (x)\right) .
\end{aligned}
$$

By making some algebraic properties of complex numbers, the general pattern coinciding with the exact solution can be established as follows:

$$
\begin{aligned}
u(x, t)= & e^{i x}+i\left(D_{x}^{\beta+1} e^{i x}-2 e^{i x}\right) \frac{t^{\alpha}}{\Gamma(1+\alpha)} \\
& +i^{2}\left[\left(D_{x}^{2 \beta+2} e^{i x}-2 D_{x}^{\beta+1} e^{i x}\right)\right] \frac{t^{2 \alpha}}{\Gamma(1+2 \alpha)} \\
& +\left[4 \sin (x) \cos (x)\left(-D_{x}^{\beta+1} \sin (x)+2 \sin (x)\right)\right. \\
& -4 i \sin (x) \cos (x)\left(D_{x}^{\beta+1} \cos (x)-2 \cos (x)\right) \\
& +\left(2 \cos ^{2}(x)+6 \sin ^{2}(x)\right)\left(D_{x}^{\beta+1} \cos (x)-2 \cos (x)\right) \\
& \left.\left.-i\left(6 \cos ^{2}(x)+2 \sin ^{2}(x)\right)\right)\left(-D_{x}^{\beta+1} \sin (x)+2 \sin (x)\right)\right] \frac{t^{2 \alpha}}{\Gamma(1+2 \alpha)}+\cdots
\end{aligned}
$$

Case 2: Eqs. (74)-(77) transform as follows:

$$
\begin{aligned}
& i v_{t}+D_{x}^{\beta+1} I_{t}^{\alpha-1} v-2\left|I_{t}^{\alpha-1} v\right|^{2} I_{t}^{\alpha-1} v=0, \\
& v(x, 0)=0 \\
& v(0, t)=I_{t}^{1-\alpha} e^{-3 i t}, \\
& v_{x}(0, t)=i I_{t}^{1-\alpha} e^{-3 i t} .
\end{aligned}
$$

To construct the approximate solution, then from Eq. (36), Eqs. (64)-(67) can be converted into an equivalent system of PDEs as follows:

$$
\begin{aligned}
& w_{t}+D_{x}^{\beta+1} I_{t}^{\alpha-1} z-2\left[\left(I_{t}^{\alpha-1} w\right)^{2}+\left(I_{t}^{\alpha-1} z\right)^{2}\right] I_{t}^{\alpha-1} z=0, \\
& z_{t}-D_{x}^{\beta+1} I_{t}^{\alpha-1} w+2\left[\left(I_{t}^{\alpha-1} w\right)^{2}+\left(I_{t}^{\alpha-1} z\right)^{2}\right] I_{t}^{\alpha-1} w=0,
\end{aligned}
$$

with the boundary conditions

$$
\begin{aligned}
& w_{0}(x, t)=I_{t}^{1-\alpha} \cos (3 t)+x I_{t}^{1-\alpha} \sin (3 t), \\
& z_{0}(x, t)=-I_{t}^{1-\alpha} \sin (3 t)+x I_{t}^{1-\alpha} \cos (3 t) .
\end{aligned}
$$

Here $\delta=1, \gamma=1, \phi(x)=0, f_{0}(x)=I_{t}^{1-\alpha} \cos (3 t), f_{1}(x)=I_{t}^{1-\alpha} \sin (3 t)$ and $g_{0}(x)=-I_{t}^{1-\alpha} \sin (3 t)$, $g_{1}(x)=I_{t}^{1-\alpha} \cos (3 t)$. Anyhow, using the RPS method, starting with the initial guessed approximations $w_{0}(x, t)=I_{t}^{1-\alpha} \cos (3 t)+x I_{t}^{1-\alpha} \sin (3 t)$ and $z_{0}(x, t)=-I_{t}^{1-\alpha} \sin (3 t)+x I_{t}^{1-\alpha} \cos (3 t)$ with the $k$ th truncated residual functions of Eq. (82) used when $j=3$ throughout the computations, the following forms for the unknown coefficients $I_{t}^{\alpha-1} f_{j}, I_{t}^{\alpha-1} g_{j}, j=0,1,2,3$, are 
obtained:

$$
\begin{aligned}
I_{t}^{\alpha-1} f_{0}(t) & =\cos (3 t), \\
I_{t}^{\alpha-1} f_{1}(t) & =\sin (3 t), \\
I_{t}^{\alpha-1} f_{2}(t) & =-D_{t}^{\alpha} \sin (3 t)+2 \cos (3 t) \\
& =\frac{-3}{2} t^{1-\alpha}\left[E_{1,2-\alpha}(3 i t)+E_{1,2-\alpha}(-3 i t)\right]+2 \cos (3 t), \\
I_{t}^{\alpha-1} f_{3}(t) & =D_{t}^{\alpha} \cos (3 t)+2\left(\sin ^{3}(3 t)+\sin (3 t) \cos ^{2}(3 t)\right) \\
& =\frac{3 i}{2} t^{1-\alpha}\left[E_{1,2-\alpha}(3 i t)-E_{1,2-\alpha}(-3 i t)\right]+2\left(\sin ^{3}(3 t)+\sin (3 t) \cos ^{2}(3 t)\right), \\
I_{t}^{\alpha-1} g_{0}(t) & =-\sin (3 t), \\
I_{t}^{\alpha-1} g_{1}(t) & =\cos (3 t), \\
I_{t}^{\alpha-1} g_{2}(t) & =-D_{t}^{\alpha} \cos (3 t)-2 \sin (3 t), \\
& =\frac{3 i}{2} t^{1-\alpha}\left[E_{1,2-\alpha}(3 i t)-E_{1,2-\alpha}(-3 i t)\right]-2 \sin (3 t), \\
I_{t}^{\alpha-1} g_{3}(t) & =-D_{t}^{\alpha} \sin (3 t)-2\left(\cos ^{3}(3 t)-\cos (3 t) \sin ^{2}(3 t)\right), \\
& =\frac{-3}{2} t^{1-\alpha}\left[E_{1,2-\alpha}(3 i t)+E_{1,2-\alpha}(-3 i t)\right]-2\left(\cos ^{3}(3 t)-\cos (3 t) \sin ^{2}(3 t)\right) .
\end{aligned}
$$

By using some algebraic properties of complex numbers, the general pattern coinciding with the exact solution can be established as follows:

$$
u(x, t)=e^{-3 i t}+i e^{-3 i t} x+3 i^{2} e^{-3 i t} \frac{x^{\beta+1}}{\Gamma(2+\beta)}+9 i^{3} e^{-3 i t} \frac{x^{\beta+2}}{\Gamma(3+\beta)}+\cdots
$$

It is clear from Figs. 7-9, the approximate solutions of Example 2 for case 1 for different orders of fractional derivatives give better results for small values $x$ and $t$. However, from Figs. 10-12 the approximate solutions of Example 2 for case 2 give a better result for $0 \leq x$, $t \leq 1$.

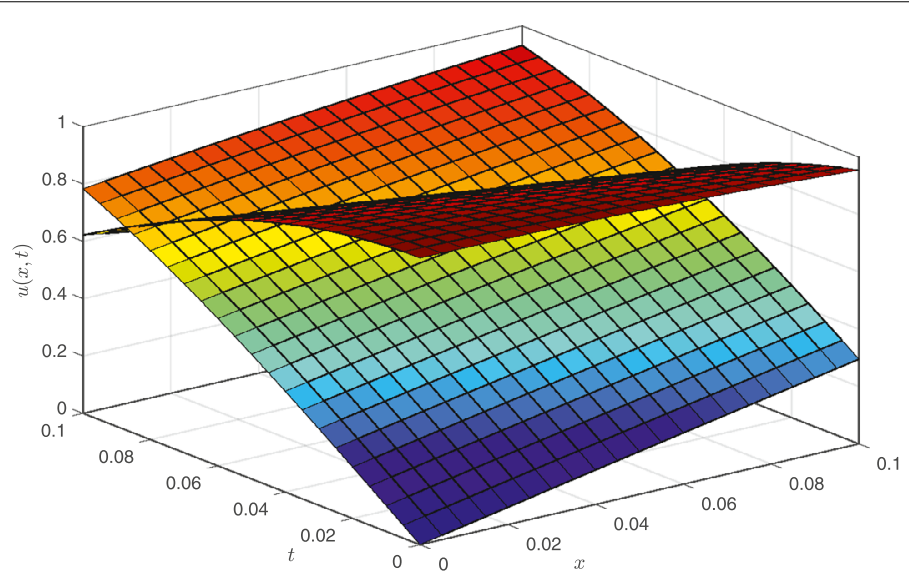

Figure 7 The approximate solution of Example 2 for Case 1 and $\alpha=\beta=1$ 


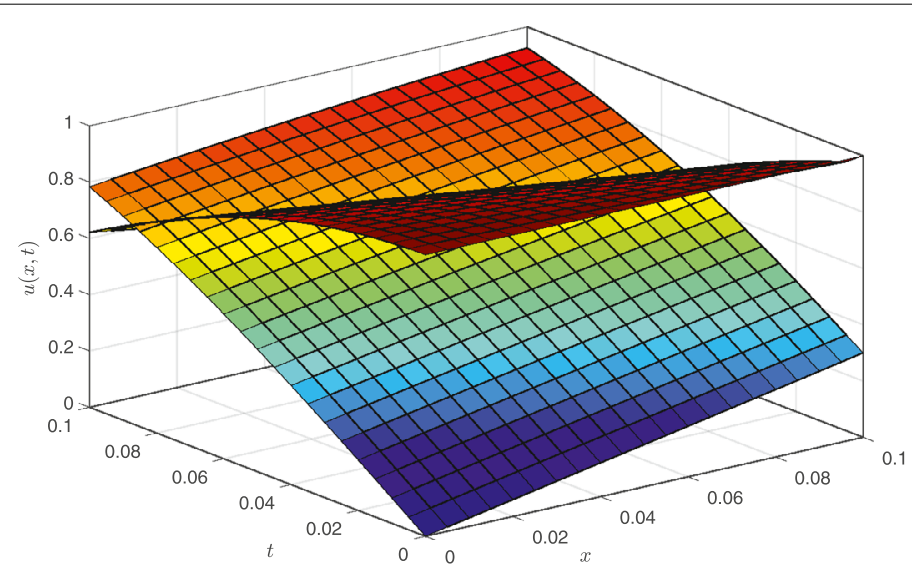

Figure 8 The approximate solution of Example 2 for Case 1 and $\alpha=\beta=0.75$

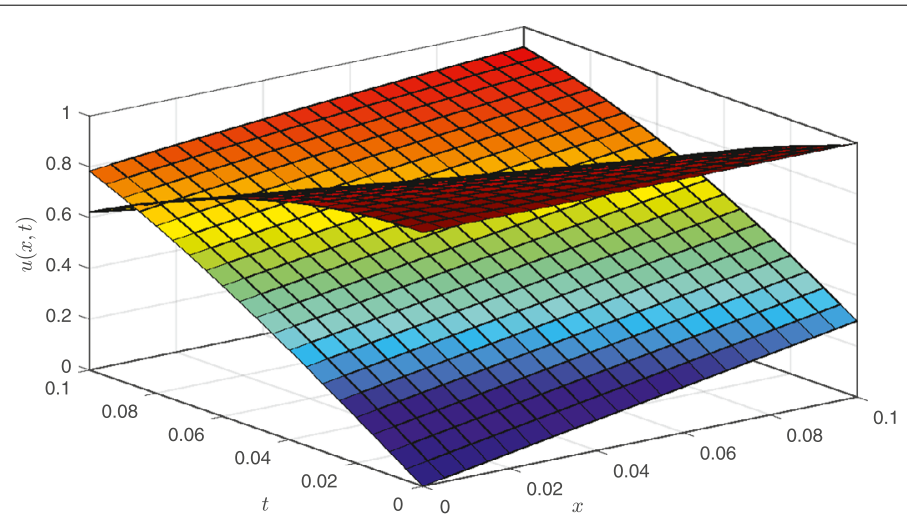

Figure 9 The approximate solution of Example 2 for Case 1 and $\alpha=\beta=0.5$

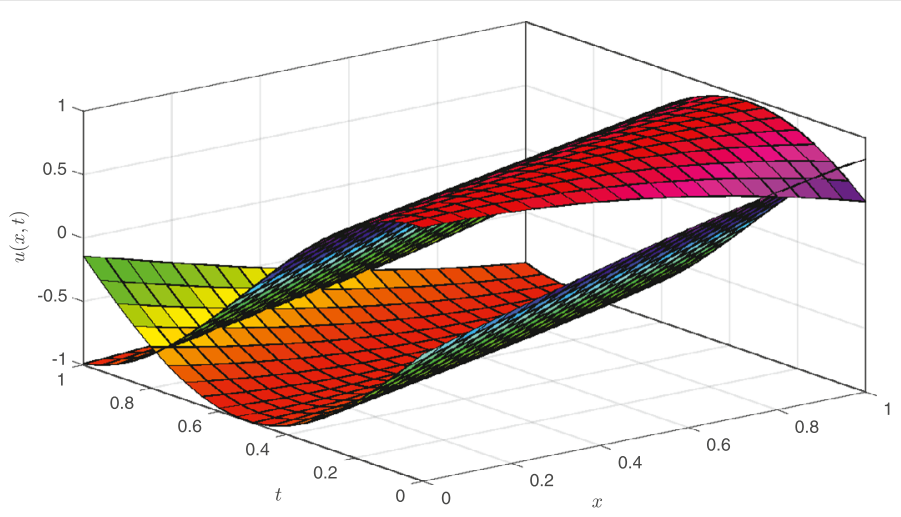

Figure 10 The approximate solution of Example 2 for Case 2 and $\alpha=\beta=1$ 


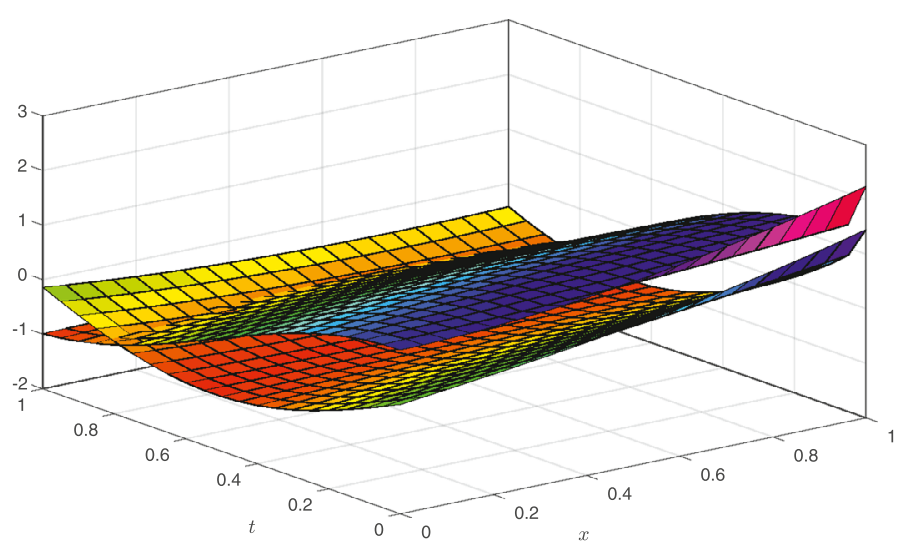

Figure 11 The approximate solution of Example 2 for Case 2 and $\alpha=\beta=0.75$

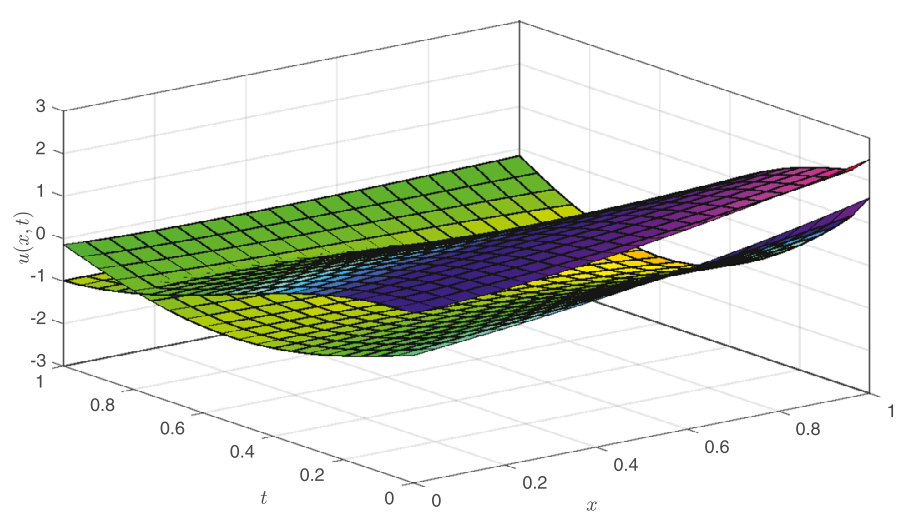

Figure 12 The approximate solution of Example 2 for Case 2 and $\alpha=\beta=0.5$

\section{Acknowledgements}

The research of the first two author was supported by Kocaeli University and the research of the third author was supported by the American University of the Middle East. The authors express their gratitude to the unknown referees for their helpful suggestions, which improved the final version of this paper.

\section{Funding}

This work is supported by the authors.

\section{Availability of data and materials}

Not applicable.

\section{Competing interests}

The authors declare that they have no competing interests.

\section{Authors' contributions}

All authors contributed equally. All authors read and approved the final manuscript.

\section{Author details}

${ }^{1}$ Department of Mathematics, Kocaeli University, Kocaeli, Turkey. ${ }^{2}$ Department of Mathematics and Statistics, American University of the Middle East, Egaila, Kuwait.

\section{Publisher's Note}

Springer Nature remains neutral with regard to jurisdictional claims in published maps and institutional affiliations. 


\section{References}

1. Komashynska, I., Al-Smadi, M., Abu Arqub, O., Momani, S.: An efficient analytical method for solving singular initial value problems of nonlinear systems. Appl. Math. Inf. Sci. 10(2), 647-656 (2016)

2. Magin, R.L., Ingo, C., Colon-Perez, L., Triplett, W., Mareci, T.H.: Characterization of anomalous diffusion in porous biological tissues using fractional order derivatives and entropy. Microporous Mesoporous Mater. 178, 39-43 (2013)

3. Cifani, S., Jakobsen, E.R.: Entropy solution theory for fractional degenerate convection-diffusion equations. Ann. Inst. Henri Poincaré, Anal. Non Linéaire 28(3), 413-441 (2011)

4. Zhang, S., Zhang, H.Q.: Fractional sub-equation method and its applications to nonlinear fractional PDEs. Phys. Lett. A 375(7), 1069-1073 (2011)

5. Mathieu, B., Melchior, P., Oustaloup, A., Ceyral, C.: Fractional differentiation for edge detection. Signal Process. 83(11), 2421-2432 (2003)

6. Mainardi, F., Raberto, M., Goreno, R., Scalas, E.: Fractional calculus and continuous-time finance II: the waiting-time distribution. Physica A 287(3-4), 468-481 (2000)

7. Caputo, M.: Linear models of dissipation whose Q is almost frequency independent: part II. Geophys. J. Int. 13(5), 529-539 (1967)

8. Podlubny, I.: Fractional Differential Equation. Academic Press, San Diego (1999)

9. Kilbas, A.A., Srivastava, H.M., Trujillo, J.J.: Theory and Applications of Fractional Differential Equations. Elsevier, Amsterdam (2006)

10. Ghanbari, B., Baleanu, D.: A novel technique to construct exact solutions for nonlinear partial differential equations. Eur. Phys. J. Plus 134(10), 1-21 (2019)

11. Yusuf, A., Inc, M., Baleanu, D.: Optical solitons with M-truncated and beta derivatives in nonlinear optics. Front. Phys. 7(126), 1-8 (2019)

12. Inc, M., Aliyu, A.l., Yusuf, A., Bayram, M., Baleanu, D.: Optical solitons to the $(n+1)$-dimensional nonlinear Schrodinger's equation with Kerr law and power law nonlinearities using two integration schemes. Mod. Phys. Lett. B 33, 19 (2019)

13. Yousef, F., Alquran, M., Jaradat, I., Momani, S., Baleanu, D.: Ternary-fractional differential transform schema: theory and application. Adv. Differ. Equ. 2019, 197, 1-13 (2019)

14. Baleanua, D., Agarwal, P., Parmare, R.K., Alqurashif, M.M., Salahshourg, S.: Extension of the fractional derivative operator of the Riemann-Liouville. J. Nonlinear Sci. Appl. 10(6), 2914-2924 (2017)

15. Morales-Delgado, V.F., Gómez-Aguilar, J.F., Saad, K.M., Khan, M.A., Agarwal, P.: Analytic solution for oxygen diffusion from capillary to tissues involving external force effects: a fractional calculus approach. Phys. A, Stat. Mech. Appl. 523, 48-65 (2019)

16. Jain, S., Agarwal, P., Kilicman, A.: Pathway fractional integral operator associated with 3m-parametric Mittag-Leffler functions. Int. J. Appl. Comput. Math. 4(5), 1-7 (2018) 115

17. Jain, S., Agarwal, P., Kıymaz, I.O., Cetinkaya, A.: Some composition formulae for the MSM fractional integral operator with the multi-index Mittag-Leffler functions. AIP Conf. Proc. 1926(1), 020020 (2018)

18. Tariboon, J., Ntouyas, S.K., Agarwal, P.: New concepts of fractional quantum calculus and applications to impulsive fractional q difference equations. Adv. Differ. Equ. 2015, 18, 1-19 (2015)

19. Ruzhansky, M., Je Cho, Y., Agarwal, P., Area, I.: Advances in Real and Complex Analysis with Applications. Springer, Singapore (2017)

20. Agarwal, P., Choib, J., Paris, R.B.: Extended Riemann-Liouville fractional derivative operator and its applications. J. Nonlinear Sci. Appl. 8(5), 451-466 (2015)

21. Agarwal, P.: Further results on fractional calculus of Saigo operators. Appl. Appl. Math. 7(2), 585-594 (2012)

22. Agarwal, P., Al-Mdallal, Q., Je Cho, Y., Jain, S.: Fractional differential equations for the generalized Mittag-Leffler function. Adv. Differ. Equ. 2018, 58, 1-8 (2018)

23. Agarwal, P., Jain, S.: Further results on fractional calculus of Srivastava polynomials. Bull. Math. Anal. Appl. 3(2), 167-174 (2011)

24. Agarwal, P., Jain, S., Mansour, T.: Further extended Caputo fractional derivative operator and its applications. Russ. J. Math. Phys. 24(4), 415-425 (2017)

25. Rekhviashvili, S., Pskhu, A., Agarwal, P., Jain, S.: Application of the fractional oscillator model to describe damped vibrations. Turk. J. Phys. 4(3), 236-242 (2019)

26. Alderremy, A.A., Saad, K.M., Agarwal, P., Aly, S., Jain, S.: Certain new models of the multi space-fractional Gardner equation. Phys. A, Stat. Mech. Appl. 2019, 123806 (2019). https://doi.org/10.1016/j.physa.2019.123806

27. Laskin, N.: Fractional Schrodinger equation. Phys. Rev. E 66, 5, 1-7 (2002)

28. Laskin, N.: Fractional quantum mechanics. Phys. Rev. E 62, 3135-3145 (2000)

29. Laskin, N.: Fractional quantum mechanics and Lévy path integrals. Phys. Lett. A 268, 298-305 (2000)

30. Laskin, N.: Fractals and quantum mechanics. Chaos 10, 780-790 (2000). https://doi.org/10.1063/1.1050284

31. Abu Arqub, O.: Series solution of fuzzy differential equations under strongly generalized differentiability. J. Adv. Res. Appl. Math. 5(1), 31-52 (2013)

32. Ford, N.J., Rodrigues, M.M., Vieira, N.: A numerical method for the fractional Schrödinger type equation of spatial dimension two. Fract. Calc. Appl. Anal. 16(2), 454-468 (2013)

33. Ashyralyev, A., Hicdurmaz, B.: On the numerical solution of fractional Schrödinger differential equations with the Dirichlet condition. Int. J. Comput. Math. 89(13-14), 1927-1936 (2012)

34. Abu Arqub, O.: Application of residual power series method for the solution of time-fractional Schrödinger equations in one-dimensional space. Fundam. Inform. 166, 87-110 (2019)

35. Zhao, Y., Cheng, D.F., Yang, X.J.: Approximation solutions for local fractional Schrödinger equation in the one-dimensional Cantorian system. Adv. Math. Phys. 2013, Article ID 291386, 1-5 (2013)

36. Kamran, A., Hayat, U., Yildirim, A., Mohyuddin, S.T.: A reliable algorithm for fractional Schrödinger equations. Walailak J. Sci. Technol. 10(4), 405-413 (2013)

37. Bibi, A., Kamran, A., Hayat, U., Mohyuddin, S.T.: New iterative method for time-fractional Schrödinger equations. World J. Model. Simul. 9(2), 89-95 (2013)

38. Naber, M.: Time fractional Schrödinger equation revisited. Adv. Math. Phys. 2013, 1-11 (2013)

39. Saxena, R.K., Saxena, R., Kalla, S.L.: Solution of space time fractional Schrödinger equation occurring in quantum mechanics. Fract. Calc. Appl. Anal. 13(2), 177-190 (2010) 
40. Wang, S., Xu, M.: Generalized fractional Schrödinger equation with space-time fractional derivatives. J. Math. Phys. 48(4), 1-10 (2007)

41. Dong, J., Xu, M.: Space-time fractional Schrödinger equation with time-independent potentials. J. Math. Anal. Appl. 344(2), 1005-1017 (2008)

42. Guo, X., Xu, M.: Some physical applications of fractional Schrödinger equation. J. Math. Phys. 47(8), 1-9 (2006)

43. Jiang, X.Y.: Time-space fractional Schrödinger like equation with a nonlocal term. Eur. Phys. J. Spec. Top. 193(1), 61-70 (2011)

Submit your manuscript to a SpringerOpen ${ }^{\circ}$ journal and benefit from:

- Convenient online submission

- Rigorous peer review

- Open access: articles freely available online

- High visibility within the field

- Retaining the copyright to your article

Submit your next manuscript at $\gg$ springeropen.com 